\title{
Core-Shell Charge Transfer In Plasmonic Fe@Ag Nanoparticles On Mgo Film
}

\author{
S. Benedetti*, I. Valenti, S. Valeri
}

Dipartimento di Scienze Fisiche Informatiche Matematiche, Università di Modena e Reggio Emilia and CNR-Istituto Nanoscienze, via Campi 213/a 41125 Modena, Italy

*Corresponding author: stefania.benedetti@unimore.it

\begin{abstract}
In this work we report the interfacial charge transfer between Fe core and Ag shell in self-organized nanoparticles on $\mathrm{MgO}$ films on $\mathrm{Mo}(001)$. Pre-deposited Fe nanoparticles organize in a square network with long range order on the oxide surface guided by the $\mathrm{MgO}$ coincidence lattice. When Ag is added, it covers the Fe nanoparticles forming a shell. By means of XPS and UPS we show that a charge transfer occurs between the Fe core and the Ag shell, determining the oxidation of part of the Fe atoms and a negative charging of the Ag shell. This is confirmed by band bending and core level shifts. As a consequence of the Fe@ Ag morphology and composition the plasmonic response of the nanoparticles is modified with respect to pure Ag nanoparticles.
\end{abstract}

\section{Introduction}

Metal nanoparticles (NPs) play a major role as catalysts in nanoscale systems, improving performances of catalytic converters, fuel cells, plasmonic devices. ${ }^{1,2,3}$ Their activity strongly depends on several contributions, like particle size, shape, exposed active sites, metal-support interaction. Within this framework an important contribution comes from the charge transfer between oxide support and NP. It was extensively reported that metal NPs deposited on thin oxide films can experience a charge transfer (both positive or negative, depending on the system) from/to the metal support or from/to oxide defects. ${ }^{4}$ This transferred charge can act directly on the adsorption rate and reaction kinetics of the adsorbates, ${ }^{5,6}$ or indirectly modifying the NP shape. ${ }^{7}$ When the NPs sustain plasmon resonances, as in the case of noble metals, the interplay between NP charging and plasmonic response must be taken into account, as it can lead to a modification in the optical properties. ${ }^{8}$ The presence of excess charges in small NPs induces a small shift of plasmon resonance, modify the dielectric properties and influences the dynamic properties of plasmon losses and hot electron injection. ${ }^{9}$ For this reason NP charging can be used to actively control the plasmon response of nanostructures and it has important consequences on their application in plasmonic devices. 
In this work we investigate the electronic properties and plasmonic response of $\mathrm{Ag}$ NPs on $\mathrm{MgO}$ film, when the NP assembly is induced by the pre-deposition of Fe seeds on the oxide surface. It has been previously shown that Fe NPs nucleate on $\mathrm{MgO}$ thin films on Mo with a preferential site on the coincidence lattice on the oxide terraces, due to oxide lattice and workfunction modulation. ${ }^{10}$ When metal NPs sit on $\mathrm{MgO}$ thin films on Mo, a spontaneous charge transfer between the metal support and the NPs occur by tunneling through the oxide. ${ }^{11,12}$ The direction of charge transfer depends on the metal/oxide system, as both negatively and positively charged NPs have been reported. When the oxide film is thick this transfer is inhibited, but other channels are active. $\mathrm{MgO}$ dislocations can act as electron traps, ${ }^{13}$ therefore adsorbates and metal NPs can positively charge when they adsorb/nucleate in their proximity. ${ }^{14,15} \mathrm{Ag}$ in particular nucleates on the dislocation network that reaches the $\mathrm{MgO}$ surface, neglecting the presence of the coincidence lattice on the terraces. ${ }^{16}$ We show here that Fe seeds induce Ag nucleation with a long range order avoiding metal intermixing and that, since Ag deposition occurs at room temperature where the alloying is known to be inhibited, Ag forms a shell on the Fe seeds. ${ }^{17}$ As a consequence of the presence of $\mathrm{Fe}$, the Ag shell negatively charges and its plasmonic response is modified.

\section{Experimental}

A Mo(100) single crystalline support has been prepared by subsequent cycles of Ar sputtering at room temperature and at $1120 \mathrm{~K}$, until a negligible quantity of $\mathrm{O}$ and $\mathrm{C}$ is found on the surface. $\mathrm{MgO}$ films on $\mathrm{Mo}(001)$ have been deposited by $\mathrm{Mg}$ thermal evaporation in $4 \times 10^{-8} \mathrm{Torr}_{2}$ partial pressure introduced through a nozzle near the Mo surface. Details on the preparation of $\mathrm{MgO}$ films on $\mathrm{Mo}(100)$ can be found elsewhere. ${ }^{18,19} \mathrm{Fe}$ and Ag nanoparticles have been deposited by thermal evaporation in ultra-high-vacuum (UHV) on the $\mathrm{MgO}$ film kept at room temperature (RT), in a base pressure of $4 \times 10^{-10}$ Torr.

X-ray photoelectron spectroscopy (XPS) measurements are performed with an Al-Ka nonmonochromatized X-ray source and a hemispherical analyzer at normal emission. Ultraviolet photoelectron spectroscopy (UPS) spectra are obtained with HeI photons. Workfunction is measured from the Secondary Electron Cutoff (SECO) exciting with HeI photons and keeping the sample biased at $-20 \mathrm{~V}$. Electron energy loss spectroscopy (EELS) measurements are performed with a primary electron beam of $100 \mathrm{eV}$ and scattered electrons are collected with the hemispherical analyzer in specular geometry at $32^{\circ}$ from surface normal. The plasmon resonances of the metal NPs have been simulated with the Granfilm code ${ }^{20}$.

Fe@ Ag NPs have been deposited on 10 ML MgO film on $\mathrm{Ag}(001)$ for comparison. MgO has been thermally evaporated in $4 \times 10^{-8}$ Torr $\mathrm{O}_{2}$ partial pressure on the support previously cleaned by 
sputtering and annealing and kept at $450 \mathrm{~K}$ during deposition. ${ }^{21,22}$

Scanning tunneling microscopy (STM) images are collected with an Omicron RT UHV AFM/STM and images are analyzed by means of WSxM. ${ }^{23}$ Grain analysis has been obtained with SPIP software.

All measurements are performed in a single UHV apparatus without breaking the vacuum.

\section{Results and discussion}

Figure 1a-d reports the STM images of the Ag nanoparticles grown on $10 \mathrm{ML} \mathrm{MgO}$ film on $\mathrm{Mo}(001)$. Before the metal deposition the coincidence lattice is visible on the $\mathrm{MgO}$ surface, as previously reported (Figure 1a). ${ }^{18,19}$ This lattice is both a morphological and electronic modulation due to the lattice mismatch and has a square periodicity of $5.5 \mathrm{~nm}$. During the deposition of $0.5 \AA$ $\mathrm{Ag}$ at room temperature the metal organizes spontaneously in form of NPs of square shape with the
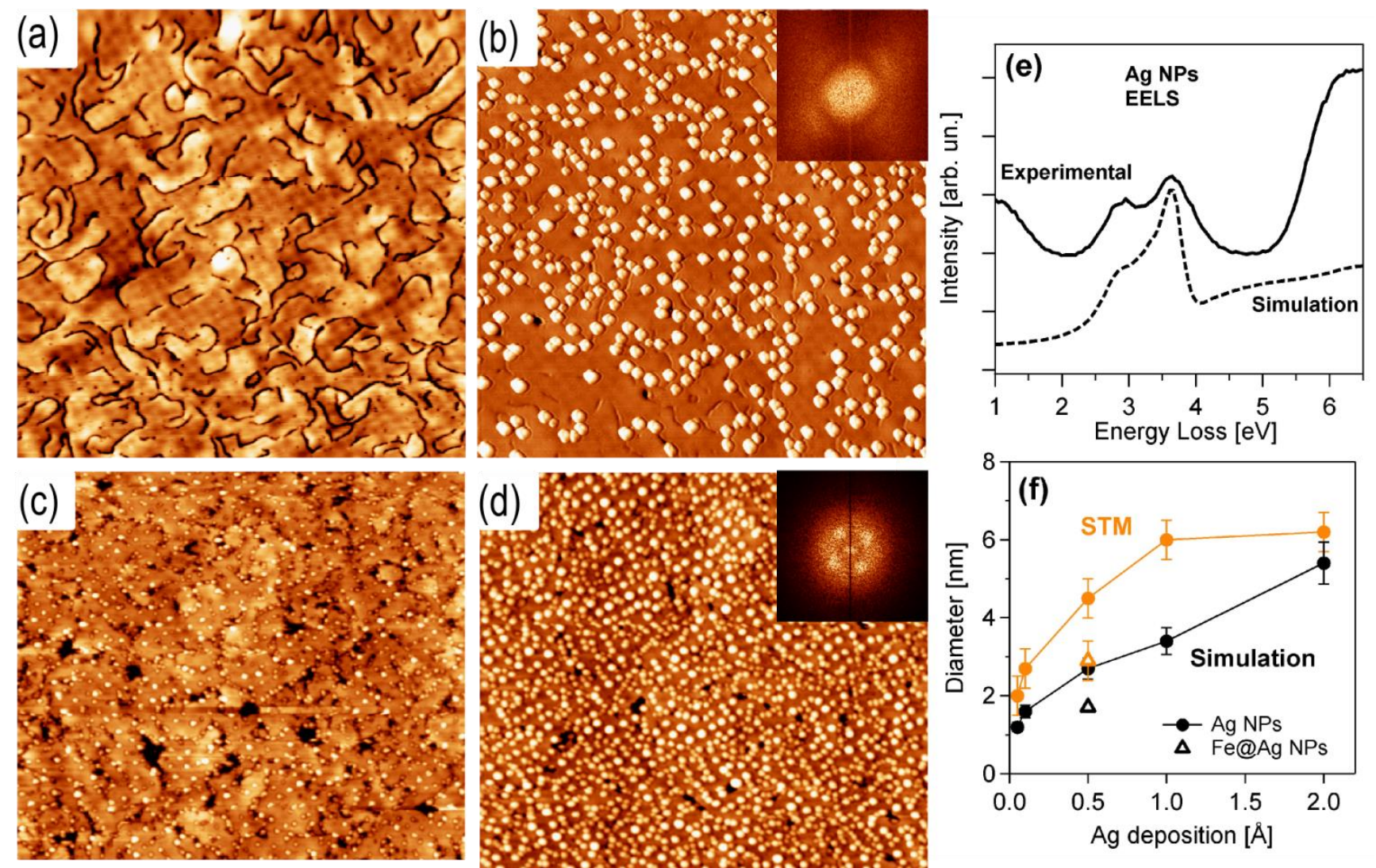

Figure 1: 100x100 nm ${ }^{2}$ STM images of (a) $10 \mathrm{ML} \mathrm{MgO}$ on $\mathrm{Mo}(001)$ (I=0.05 nA, U=3.9 V), (b) 0.5 $\AA \mathrm{Ag}$ on $10 \mathrm{ML} \mathrm{MgO}(\mathrm{I}=0.04 \mathrm{nA}, \mathrm{U}=3.8 \mathrm{~V})$, (c) $0.4 \AA$ A Fe on $10 \mathrm{ML} \mathrm{MgO}(\mathrm{I}=0.03 \mathrm{nA}, \mathrm{U}=3.2 \mathrm{~V}),(\mathrm{d})$ $0.5 \AA \mathrm{Ag} / 0.4 \AA \mathrm{Fe} / 10 \mathrm{ML} \mathrm{MgO}(\mathrm{I}=0.06 \mathrm{nA}, \mathrm{U}=3.2 \mathrm{~V})$. Insets of $(\mathrm{b}, \mathrm{d})$ report the FFT of the respective images.(e) EELS spectra and Granfilm simulation for Ag NPs in (b); (f) NP diameter as obtained by STM grain analysis (orange) and EELS simulations (black).

[100] sides parallel to the $\mathrm{MgO}[100]$ surface direction, with average diameter of $4.5 \mathrm{~nm}$ and height $1.6 \mathrm{~nm}$ (Figure 1b). NPs nucleate on the dislocations of the oxide film (black cracks in Figure 1a), 
while they neglect the presence of the coincidence lattice. ${ }^{16}$ On the contrary Fe NPs nucleate both on the dislocations and on the coincidence lattice, showing a partial long range order, with average diameter of $1.8 \mathrm{~nm}$ and height $0.5 \mathrm{~nm}$ (Figure 1c). ${ }^{10}$ If we deposit Ag after the formation of Fe NPs, they maintain the ordering of the Fe NPs, as evident in the FFT, with a periodicity of $5.5 \mathrm{~nm}$ (Figure 1d). The density of the NPs remains almost constant before and after Ag deposition $\left(4.8 \times 10^{12} \mathrm{~cm}^{-2}\right.$ for Fe NPs, $5.3 \times 10^{12} \mathrm{~cm}^{-2}$ for $\left.\mathrm{Ag} / \mathrm{Fe}\right)$, while the diameter increases from 1.8 to 2.9 $\mathrm{nm}$ and the height from 0.5 to $0.7 \mathrm{~nm}$. Fe-Ag alloying is not favored up to the melting of the two metals, ${ }^{17}$ and since deposition of low-energy atoms occurs at room temperature, we do not expect any intermixing. Therefore we can tentatively conclude that $\mathrm{Ag}$ nucleates on the pre-formed $\mathrm{Fe}$ NPs, covering them and forming Fe@Ag core@shell NPs. Diameters can be estimated from grain analysis of STM images or from the simulation with Granfilm code of the plasmonic response of Ag NPs in Electron Energy Loss Spectra (EELS, Figure 1e). Taking NP height from STM and simulating EELS of Ag on MgO we can obtain the real NP diameter and shape. Plasmon resonance energies strictly depend on the NP morphology and we have exploited Granfilm simulation of EELS spectra to determine the NPs hemispherical shape and the actual diameters. Figure 1f reports the comparison of diameters as obtained by STM grain analysis and EELS simulations. This in accordance with previous experimental works reporting truncated spheres, approximating predicted truncated fcc octahedra in (100) epitaxy. ${ }^{24,25,26}$ Optimization of particle geometry based on comparison with simulations leads to an average NP diameter of $2.7 \mathrm{~nm}$ for nominally $0.5 \AA \mathrm{Ag}$ on $\mathrm{MgO}$ film. This indicates a correction factor on diameters between the two curves in Figure 1f of 1.7 (due to tip convolution). This deviates only for the point at $2 \AA$, where both the STM height is not easily determined and a larger error is introduced in EELS simulation. More details concerning the two estimations are given in the Supporting Information. Using this correction factor we can correct the diameter of Fe@ Ag NPs to $1.7 \mathrm{~nm}$. 
Combining the obtained morphological information with XPS we can better determine the composition of the NPs, adding information concerning possible interfacial interaction. For this reason we have investigated the electronic properties of the system by means of XPS and UPS. The Fe2p XPS lineshape of Fe NPs on 10 ML MgO shows a clear metallic state (Figure 2a). A clear asymmetry (as compared to bulk Fe) is present on the high binding energy side $(710 \mathrm{eV})$, that can be due to the reduced dimensionality. ${ }^{27}$ This asymmetry does not modify when the system is exposed to the partial pressure present in the deposition chamber when the Ag evaporator is
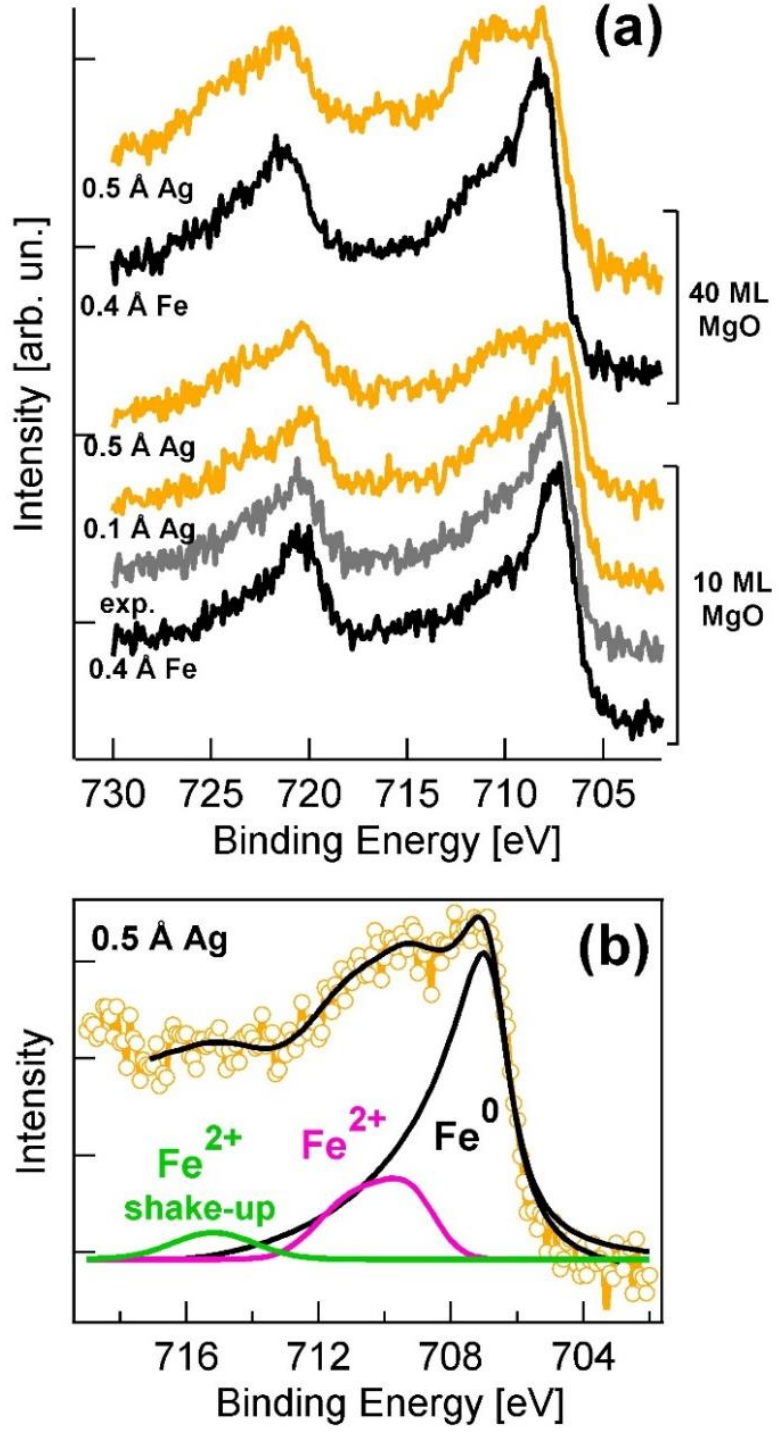

Figure 2: (a) Fe2p XPS spectra of $0.4 \AA \mathrm{Fe}$ on 10 (lower part) and $40 \mathrm{ML} \mathrm{MgO} \mathrm{(upper}$ part) before (black spectra) and after Ag deposition (orange spectra). Grey curve reports the peak after Fe deposition on $\mathrm{MgO}$. (b) Fit of the $\mathrm{Fe} 2 \mathrm{p}_{3 / 2}$ measured after Ag deposition. switched on (Figure 2a, grey spectrum).

However as soon as Ag is deposited on top, a second component appears on the high binding energy side, compatible with $\mathrm{Fe}^{2+}$ ions. This component increases with increasing deposited Ag quantity and is slightly more intense for thicker $\mathrm{MgO}$ films. To establish the amount and trend in Fe oxidation we have performed a deconvolution of Fe2p3/2 peak (Figure $2 b$ ) with tabulated peak positions and intensity ratios of $\mathrm{Fe}^{2+}$ (as in $\mathrm{FeO}$ ) and shakeup satellite, while for $\mathrm{Fe}^{0}$ we have considered the Fe lineshape measured before $\mathrm{Ag}$ deposition (that takes into account the low dimensionality effects). ${ }^{28} \mathrm{With}$ this quantification, $\mathrm{Fe}^{2+} / \mathrm{Fe}^{0}$ area ratio after $0.1 \AA$ deposition is 0.15 , for $0.5 \AA \mathrm{Ag}$ is 0.25 , on 40 $\mathrm{ML} \mathrm{MgO}$ is 0.33 .

Given the NP diameters reported in Figure 1f, we have quantitatively estimated the thickness of the core and shell by means of a model for XPS attenuation applied to NPs. ${ }^{29}$ The model starts from a planar configuration calculation and assuming a correction topofactor for the spherical geometry allows to determine the NP shell thickness. We have applied this model to the $\mathrm{Fe}^{2+} / \mathrm{Fe}^{0}$ intensity ratio obtained by the $\mathrm{Fe} 2 \mathrm{p}$ 
deconvolution to estimate the thickness of the $\mathrm{Fe}^{2+}$ shell inside the core. Then from the $\mathrm{Ag} 3 \mathrm{~d} / \mathrm{Fe} 2 \mathrm{p}$ intensity ratio we have determined the external Ag shell thickness. We obtain that the Ag shell is $0.34 \mathrm{~nm}$ thick, while the core is reasonably divided in a Fe ${ }^{2+}$ shell of $0.2 \mathrm{~nm}$ on a central $\mathrm{Fe}^{0}$ nucleus of $0.11 \mathrm{~nm}$.

To clarify the origin of the Fe oxidation we have studied the electronic properties of the system combining XPS and UPS. Mg and O lineshapes do not change with $\mathrm{Fe}$ and $\mathrm{Ag}$ deposition. Core level peak position has been determined by a fit with Gaussian or Voigt function, while VB edge position is obtained by the crossing point between the linear fit of the leading edge of the valence band and the background measured in UPS spectra. A shift of the valence band (VB) edge and of core level peaks of the oxide film and the Ag NPs has been observed. This indicates a band bending $V_{b b}$ occurring upon metal deposition (Figure 3). We report the shift of the Mg1s surface sensitive core level peak with respect to the clean $\mathrm{MgO}$ film signal in Figure 3a. The peak of the $10 \mathrm{ML}$ film shifts to lower binding energy (upward bending) when $\mathrm{Ag}$ alone is deposited. For Ag quantities below $1 \AA$ A however shifts are negligible and correspondingly also valence band edge is almost constant (Figure 3b). Correspondingly we obtain an increase in

workfunction $\Delta \varphi$ as measured by the low-energy onset of secondary electron distribution in the UPS spectrum and the subsequent increase in ionization energy (calculated as $\left.\Delta I=\Delta \varphi+\Delta V_{b b}\right)^{30}$ of $+0.3 \mathrm{eV}$ are observed for $0.5 \AA \mathrm{Ag}$ as compared with the pure $\mathrm{MgO}$ surface. of $+0.3 \mathrm{eV}$ are observed for $0.5 \AA \mathrm{Ag}$ as compared with the pure $\mathrm{MgO}$ surface. This indicates the

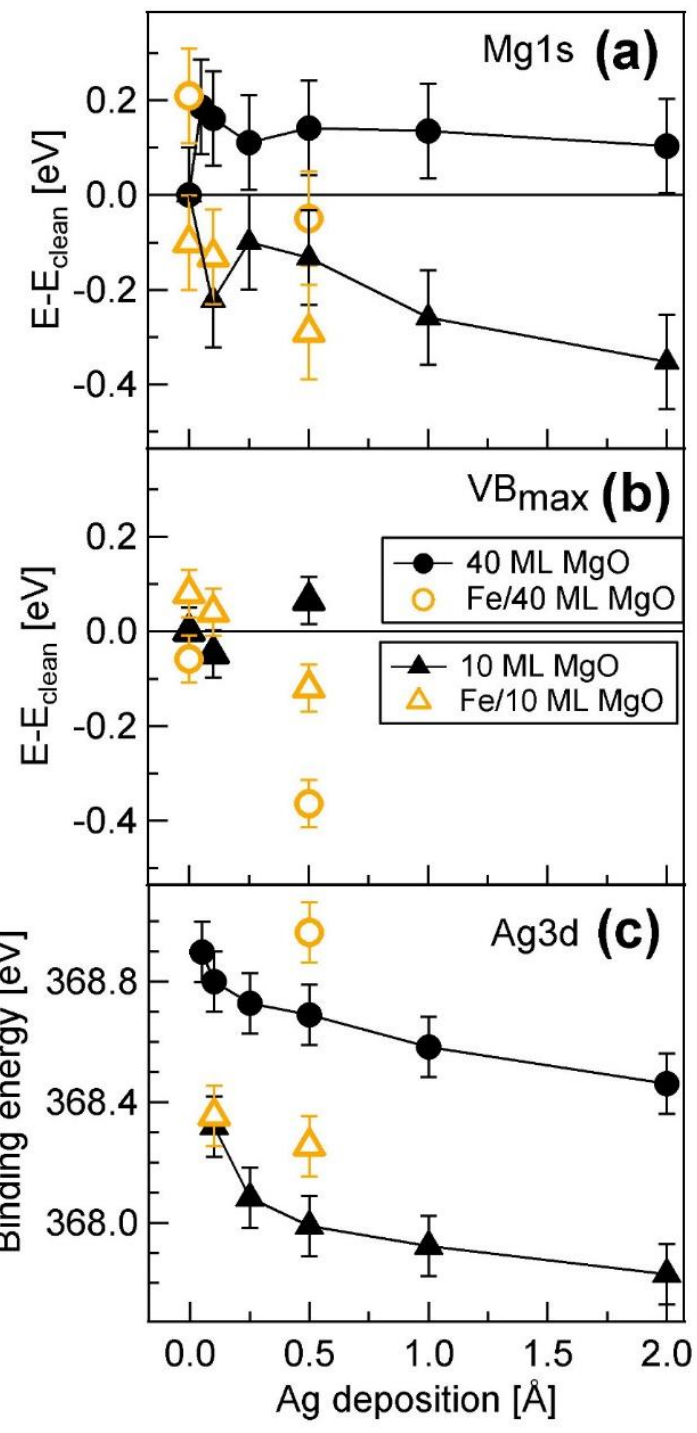

Figure 3: Variations with Ag deposited quantity of (a) Mg1s and (b) valence band edge position, determined as difference with respect to the clean oxide film. (c) Binding energy position of $\mathrm{Ag} 3 \mathrm{~d} 5 / 2$ peak on $10 \mathrm{ML}$ and $40 \mathrm{ML}$ oxide film (full markers) and after 0.4 $\AA$ Fe deposition (empty markers). presence of a negative surface dipole, compatible with a charge transfer from the Mo support to the NPs, as it occurs for Au NPs on MgO film. ${ }^{12,31}$ When Fe core is added to the NPs, both the Mg1s 
and VB edge shift increases indicating a stronger upward bending, indicating a larger charge transfer $(\Delta \varphi=+0.6 \mathrm{eV}, \Delta \mathrm{I}=+0.5 \mathrm{eV}$ for $0.5 \AA \mathrm{Ag})$. Therefore Fe oxidation is due to the transfer of electrons from the external part of the Fe core to the $\mathrm{Ag}$ shell (and not to $\mathrm{O}$ inclusion, contamination from residual gas has been excluded). When $\mathrm{Ag}$ NPs are grown on $40 \mathrm{ML} \mathrm{MgO}$, the bending is downward, indicating a positive charging of the Ag NPs (without Fe). Here tunneling through the oxide is inhibited, therefore the charges are trapped in the oxide dislocations. ${ }^{13,15,14}$ When $\mathrm{Fe}$ is added the bending inverts due to charges moving to the external Ag shell, inverting the surface dipole, and peaks move to lower binding energy. A small shift is present also when Fe alone is deposited, indicating a small charge transfer also from Fe NPs to MgO. Therefore the negative charge transfer to $\mathrm{Ag}$ is even more evident in this case.

The charging is evident also from the Ag3d5/2 peak position, reported in Figure 3c. The peak shifts to lower BE with increasing Ag quantity (without Fe). This decrease of Ag electron binding energy at higher deposition is related to the increase in NP size, as reported in Figure $1 \mathrm{f}^{32}$ When $\mathrm{Fe}$ is present in the core, the BE increases by $0.25 \mathrm{eV}$ at $0.5 \AA \mathrm{Ag}$. These Fe@ Ag NPs have a diameter comparable to those obtained by deposition of $0.25 \AA$ pure Ag (Figure 1f), while the Ag3d binding energy is still higher than $0.25 \AA \mathrm{Ag}$. Therefore this increase cannot cannot be entirely explained by the reduced dimensionality. On the contrary it can be related to the presence of negative charges, since in bulk Ag the Ag 3d binding energy at higher oxidation state is lower than in the metallic state due to a large extra-atomic relaxation energy contribution, which dominates over the positive shift in the initial state. ${ }^{33}$

The origin of $\mathrm{Fe}$ oxidation is therefore a charge transfer from Fe to Ag due to the higher $\mathrm{Ag}$ electron affinity, particularly important at low dimensionality. When Ag adatom is put in contact with (or embedded in) Fe surface, a shift in the d-bands is predicted, but no modification of the band occupancy is predicted. ${ }^{34}$ An hybridization at the interface between Fe and Ag states is expected, however this does not explain the charge transfer. Contrary to the charge transfer mechanisms playing a role without $\mathrm{Fe}$, here the effect is not fully related to the presence of the Mo support or the $\mathrm{MgO}$ dislocations, since the same effect is evident also on Fe@ $\mathrm{Ag}$ NPs on MgO film on $\mathrm{Ag}(001)$, even if to a smaller extent, where charge transfer from the metal support should be less favored. ${ }^{35}$ It is therefore a general effect present in these bimetallic NPs. Part of the contribution can additionally come from the metal support or from the $\mathrm{MgO}$ dislocations and sum to the main effect. Given the NPs size obtained from STM and XPS quantification reported before, we can conclude that, supposing every oxidized Fe atom donates 2 electrons, 0.6 electrons are donated to every $\mathrm{Ag}$ shell atom in the $0.5 \mathrm{~A} \mathrm{Ag}$ deposition, while 0.1 e/at for $0.1 \mathrm{~A} \mathrm{Ag}$ deposition. It is known that bulk 
tabulated values of binding energies in XPS spectra does not always match atomic oxidation states in low dimensional systems. However this rough estimation is consistent with numbers reported in literature. ${ }^{31,35}$
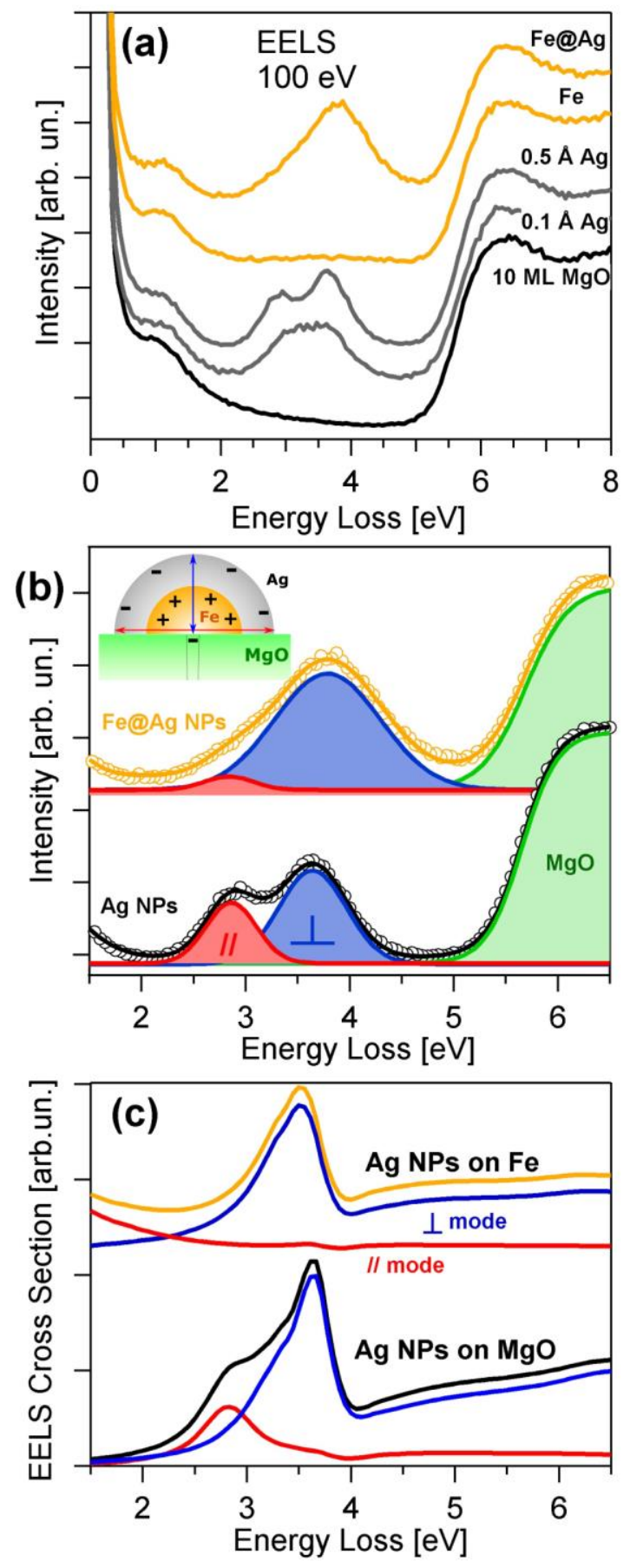

Figure 4. (a) EELS spectra of Ag and $\mathrm{Fe} @ \mathrm{Ag}$ NPs on $10 \mathrm{ML} \mathrm{MgO} / \mathrm{Mo}(001)$. (b) Fit of the plasmon modes for Ag and Fe@Ag NPs. (c) EELS cross section of $\mathrm{Ag}$ NPs on $\mathrm{MgO}$ and on Fe simulated with Granfilm code.
The presence of the Fe core has consequences on the plasmon response of the system (Figure 4). EELS spectra of Ag NPs on MgO have two main peaks at about 2.8 and $3.6 \mathrm{eV}$, assigned to the plasmonic modes respectively parallel and perpendicular to the substrate surface (Figure 4a). ${ }^{24} \mathrm{~A}$ small structure at about $1 \mathrm{eV}$ is related to the Mo support, while the interband transition edge of $\mathrm{MgO}$ is located at about $5.5 \mathrm{eV}$. Figure $4 \mathrm{~b}$ reports a fit of the Ag NPs spectra with two Gaussian peaks for the two plasmon modes and a Fermi-Dirac distribution for the $\mathrm{MgO}$ interband transition. ${ }^{36}$ A more detailed description of the EELS spectra of Ag NPs is reported in the Supporting Information. When the Fe core is present, the deconvolution of the two plasmon modes is less evident and the perpendicular mode seems dominating. To clarify if the change in the plasmon resonances can be assigned to the modified dielectric environment or if can be related to the important charge transfer, we have compared the results with the simulation of the EELS cross section by means of Granfilm code (Figure 4c). Here we have assumed hemispherical NPs of $1.7 \mathrm{~nm}$ diameter placed on a Fe surface to simulated the dielectric interface between $\mathrm{Ag}$ and Fe. Here simulation shows a strong blueshift of the parallel mode that overlaps to the perpendicular mode, determining a superposition in a unique broad peak at around $3.5 \mathrm{eV}$, compatible with the 
experimental spectra. The small contribution at $2.8 \mathrm{eV}$ that is visible in the fit for Fe@ $\mathrm{Ag}$ is due to the presence of Ag NPs nucleated without Fe core, as evidenced from the slightly larger NP density after Ag deposition on Fe. From these results a variation in the plasmonic response of the NPs due to the presence of $\mathrm{Fe}$ is evident. It is more difficult to assign an effect to the influence of charging due to the peak broadening. Further ab-initio theoretical calculations could clarify the role of this possible contribution.

\section{Conclusions}

In conclusion we have shown that when $\mathrm{Ag}$ is deposited on Fe nanoparticles on $\mathrm{MgO} / \mathrm{Mo}(001)$, they replicate the ordering of Fe. Combining STM, EELS and XPS we have demonstrated that Ag covers the Fe NPs, forming a shell around them. As soon as the Ag shell is added a charge transfer occurs from $\mathrm{Fe}$ to $\mathrm{Ag}$, leaving part of the Fe atoms in an oxidized state. At the same time bands bend upward in response to the presence of a surface negative dipole in the vicinity. Part of the negative charges transferred to Ag come from Mo support by tunneling through the oxide film, inducing an overall shift of the levels and of $\mathrm{Ag} 3 \mathrm{~d}$ states. This effect is not limited to the $\mathrm{Fe} @ \mathrm{Ag} / \mathrm{MgO} / \mathrm{Mo}$ but seems to be more general, as it is present also changing the metal support for $\mathrm{MgO}$. As a consequence of the presence of Fe, NPs modify their plasmonic resonances. These change from two non-degenerate plasmon modes (due to the hemispherical particle morphology and to the presence of the substrate) to an almost degenerate broad mode induced by the vicinity of Fe. The presence of excess charges on the particle surface and the modifications induced in their electronic and optical properties could have important consequences on their catalytic activity and can reveal as possible tool to tune their response.

\section{Acknowledgements}

Authors would like to acknowledge the financial support by the Italian MIUR through the PRIN Project 2015CL3APH - NEWLI and by Università di Modena e Reggio Emilia through the FAR project "Innovative (oxide-based) materials and methods for fuel cell electrodes implementation".

Supporting Information: Determination of Ag NPs diameter as a function of metal deposition through a comparison between STM and EELS (both experimental and simulated); the comparison of Fe@Ag NPs Fe2p lineshape on different substrates. This material is available free of charge via the Internet at http://pubs.acs.org. 


\section{References}

1. Robatjazi, H.; Bahauddin, S. M.; Doiron, C.; Thomann, I., Direct Plasmon-Driven Photoelectrocatalysis. Nano Lett. 2015, 15, 6155-6161.

2. Debe, M. K., Electrocatalyst Approaches and Challenges for Automotive Fuel Cells. Nature 2012, 486, 43-51.

3. An, K.; Somorjai, G. A., Nanocatalysis I: Synthesis of Metal and Bimetallic Nanoparticles and Porous Oxides and Their Catalytic Reaction Studies. Catal. Lett. 2015, 145, 233-248.

4. Pacchioni, G., Electronic Interactions and Charge Transfers of Metal Atoms and Clusters on Oxide Surfaces. Phys. Chem. Chem. Phys. 2013, 15, 1737-1757.

5. $\quad$ Lin, X.; Yang, B.; Benia, H. M.; Myrach, P.; Yulikov, M.; Aumer, A.; Brown, M. A.; Sterrer, M.; Bondarchuk, O.; Kieseritzky, E.; et al., Charge-Mediated Adsorption Behavior of CO on MgO-Supported Au Clusters. J. Am. Chem. Soc. 2010, 132, 7745-7749.

6. Jiang, D.; Wang, W.; Sun, S.; Zhang, L.; Zheng, Y., Equilibrating the Plasmonic and Catalytic Roles of Metallic Nanostructures in Photocatalytic Oxidation over Au-Modified CeO2. ACS Catal. 2015, 5, 613-621.

7. Shao, X.; Prada, S.; Giordano, L.; Pacchioni, G.; Nilius, N.; Freund, H. J., Tailoring the Shape of Metal Ad-Particles by Doping the Oxide Support. Angew. Chem. Int. Ed. 2011, 50, 1152511527.

8. Choi, H.; Chen, W. T.; Kamat, P. V., Know Thy Nano Neighbor. Plasmonic versus Electron Charging Effects of Metal Nanoparticles in Dye-Sensitized Solar Cells. ACS Nano 2012, 6, 44184427.

9. Herrera, M. Z.; Aizpurua, J.; Kazansky, A. K.; Borisov, A. G., Plasmon Response and Electron Dynamics in Charged Metallic Nanoparticles. Langmuir 2016, 32, 2829-2840.

10. Benedetti, S.; Stavale, F.; Valeri, S.; Noguera, C.; Freund, H. J.; Goniakowski, J.; Nilius, N., Steering the Growth of Metal Ad-particles via Interface Interactions Between a MgO Thin Film and a Mo Support. Adv. Func. Mater. 2013, 23, 75-80.

11. Giordano, L.; Baistrocchi, M.; Pacchioni, G., Bonding of Pd, Ag, and Au Atoms on $\mathrm{MgO}(100)$ Surfaces and MgO/Mo(100) Ultra-Thin Films: A Comparative DFT Study. Phys. Rev. B 2005, 72, 115403.

12. Sterrer, M.; Risse, T.; Pozzoni, U. M.; Giordano, L.; Heyde, M.; Rust, H.-P.; Pacchion, G.; Freund, H.-J., Control of the Charge State of Metal Atoms on Thin MgO Films. Phys. Rev. Lett. 2007, 98, 096107.

13. McKenna, K. P.; Shluger, A. L., Electron-Trapping in Polycrystalline Materials with Negative Electron Affinity. Nat. Mater. 2008, 7, 859-862.

14. Benia, H. M.; Myrach, P.; Gonchar, A.; Risse, T.; Nilius, N.; Freund, H. J., Electron Trapping in Misfit Dislocations of MgO Thin Films. Phys. Rev. B 2010, 81, 241415.

15. Benedetti, S.; Nilius, N.; Myrach, P.; Valenti, I.; Freund, H. J.; Valeri, S., Spontaneous Oxidation of Mg Atoms at Defect Sites in an MgO Surface. J. Phys. Chem. C 2011, 115, 36843687.

16. Benedetti, S.; Myrach, P.; di Bona, A.; Valeri, S.; Nilius, N.; Freund, H. J., Growth and Morphology of Metal Particles on $\mathrm{MgO} / \mathrm{Mo}(001)$ : A Comparative STM and Diffraction Study. Phys. Rev. B 2011, 83, 125423.

17. Kubaschewski, O., Iron-Binary Phase Diagrams. Springer: Berlin, 1982.

18. Benedetti, S.; Benia, H. M.; Nilius, N.; Valeri, S.; Freund, H. J., Morphology and Optical Properties of MgO Thin Films on Mo(001). Chem. Phys. Lett. 2006, 430, 330-335.

19. Benedetti, S.; Torelli, P.; Valeri, S.; Benia, H. M.; Nilius, N.; Renaud, G., Structure and Morphology of Thin MgO Films on Mo(001). Phys. Rev. B 2008, 78, 195411.

20. Lazzari, R.; Simonsen, I., GRANFILM: a Software for Calculating Thin-Layer Dielectric Properties and Fresnel Coefficients. Thin Solid Films 2002, 419, 124-136. 
21. Schintke, S.; Messerli, S.; Pivetta, M.; Patthey, F.; Libjoulle, L.; Stengel, M.; Vita, A. d.; Schneider, W. D., Insulator at the Ultrathin Limit: $\mathrm{MgO}$ on $\mathrm{Ag}(001)$. Phys. Rev. Lett. 2001, 87, 276801.

22. Valeri, S.; Altieri, S.; Bona, A. d.; Luches, P.; Giovanardi, C.; Moia, T. S., ThicknessDependent Strain in Epitaxial MgO Layers on Ag(001). Surf. Sci. 2002, 507, 311-317.

23. Horcas, I.; Fernández, R.; Gómez-Rodríguez, J. M.; Colchero, J.; Gómez-Herrero, J.; Baró, A. M., WSXM: A Software for Scanning Probe Microscopy and a Tool for Nanotechnology. Rev. Sci. Instrum. 2007, 78, 013705.

24. Lazzari, R.; Jupille, J.; Layet, J.-M., Electron-Energy-Loss Channels and Plasmon Confinement in Supported Silver Particles. Phys. Rev. B 2003, 68, 045428.

25. Myrach, P.; Nilius, N.; Freund, H. J., Photon Mapping of Individual Ag Particles on $\mathrm{MgO} / \mathrm{Mo}(001)$. Phys. Rev. B 2011, 83, 035416.

26. Goniakowski, J.; Mottet, C., Simulation of the Growth of Metal Nanoclusters on the MgO(100) Surface. Phys. Rev. B 2010, 81, 155443.

27. Luches, P.; Benedetti, S.; Liberati, M.; Boscherini, F.; Pronin, II; Valeri, S., Absence of Oxide Formation at the Fe/MgO(001) Interface. Surf. Sci. 2005, 583, 191-198.

28. Grosvenor, A. P.; Kobe, B. A.; Biesinger, M. C.; McIntyre, N. S., Investigation of Multiplet Splitting of Fe 2p XPS Spectra and Bonding in Iron Compounds. Surf. Interface Anal. 2004, 36, 1564-1574.

29. Shard, A. G., A Straightforward Method For Interpreting XPS Data From Core-Shell Nanoparticles. J. Phys. Chem. C 2012, 116, 16806-16813.

30. Luth, H., Surface and Interfaces of Solids. Springer Verlag: 1992; Vol. 15.

31. Torelli, P.; Giordano, L.; Benedetti, S.; Luches, P.; Annese, E.; Valeri, S.; Pacchioni, G., Xray Photoemission Study of the Charge State of Au Nanoparticles on Thin MgO/Fe(001) Films. J. Phys. Chem. C 2009, 113, 19957-19965.

32. Fu, Q.; Wagner, T., Interaction of Nanostructured Metal Overlayers with Oxide Surfaces. Surf. Sci. Rep. 2007, 62, 431-498.

33. Bao, X.; Wild, U.; Muhler, M.; Pettinger, B.; Schloegl, R.; Ertl, G., Coadsorption of Nitric Oxide and Oxygen on the Ag(110) Surface. Surf. Sci. 1999, 425, 224-232.

34. Ruban, A.; Hammer, B.; Stoltze, P.; Skriver, H. L.; Nørskov, J. K., Surface Electronic Structure and Reactivity of Transition and Noble Metals. J. Mol. Catal. A: Chemical 1997, 115, 421-429.

35. Sicolo, S.; Giordano, L.; Pacchioni, G., Adsorption of Late Transition Metal Atoms on $\mathrm{MgO} / \mathrm{Mo}(100)$ and $\mathrm{MgO} / \mathrm{Ag}(100)$ Ultrathin Films: A Comparative DFT Study. J. Phys. Chem. C 2009, 113, 16694-16701.

36. Visikovskiy, A.; Mitsuhara, K.; Hazama, M.; Kohyama, M.; Kido, Y., The Atomic and Electronic Structures of NiO(001)/Au(001) Interfaces J. Chem. Phys. 2013, 139, 144705. 
TOC Graphic

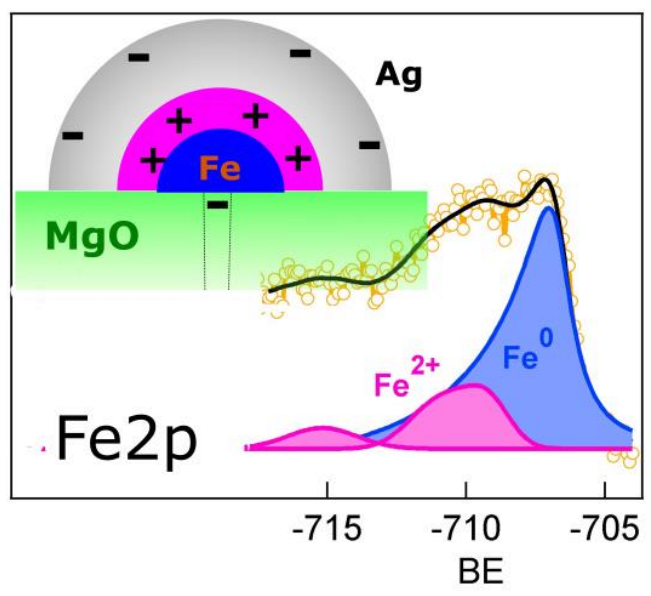

